\title{
Successful management of daytime enuresis using alarm procedures: a randomly controlled trial
}

\author{
S HALLIDAY, S R MEADOW, AND I BERG \\ Department of Paediatrics and Child Health, St James's University Hospital, Leeds
}

SUMMARY Forty four children with daytime wetting were included in a randomly controlled trial of two alarm devices, a contingent one that sounded when wetting occurred and a non-contingent one that went off from time to time unrelated to wetting events. A quota allocation system ensured comparability between treatment groups. Two thirds responded to an alarm by becoming dry. The non-contingent alarm produced as good a response as the contingent one and is recommended for routine use in children with diurnal enuresis. Twenty three per cent of those who responded to treatment relapsed up to two years after completion of the trial.

Various methods of management have been found to be effective in the treatment of nocturnal enuresis, particularly the use of the pad and bell alarm device. Unfortunately, there has not yet been comparable success in dealing with diurnal enuresis. $^{1-3}$ The enuresis alarm for bed wetting provides a loud auditory stimulus immediately after the occurrence of the wetting event. It is possible to produce a similar effect for daytime wetting using a pants alarm.

This study was set up to evaluate a contingent alarm system for the treatment of diurnal enuresis using a randomly controlled trial. Children who did not receive the device being tested were managed with what seemed to be an identical alarm but which in fact merely emitted a similar loud tone about every two hours, whether wetting had occurred or not.

\section{Patients and methods}

Children aged 5 to 15 attending a children's enuresis clinic were selected for inclusion in the trial. Daytime wetting had to be sufficiently common and severe to make it the main problem complained about by the mother. For most of the children the pants became so wet each day that urine soaked through to trousers or skirt; it was rare, however, for there to be a puddle on the chair seat or floor. About two thirds of the children were referred by consultant colleagues (tertiary referral) and the other third by general practitioners or clinical medical officers. Any children identified as having severe urinary tract abnormality were excluded; urinary tract infections were treated before the child was included in the study. Children with very severe social or psychological problems that made them unsuitable for routine management in a paediatric outpatient clinic were also excluded. Nevertheless, the sample of cases included in the study was virtually a consecutive series of children presenting with troublesome daytime wetting; only two were excluded because of being too disturbed for paediatric management.

Fifty cases were collected, but in one instance the family refused to participate, in another the child developed diabetes mellitus, and for four others treatment was not thought to be necessary as there was a period of at least seven days without wetting during the baseline period. Therefore, 44 children were included in the trial and allocated to one of the two treatment groups.

The mean (SD) age of those included was 8.5 $(2.1)$ years (range 5.0 to 13.0 years), and 35 were girls. Social class on the Registrar General's classification was 15 in classes I or II, 22 in class III, and seven in classes IV or V. Five of the children were not living with both natural parents. Birth order was about average for the general population. The mean (SE) Slater's index ${ }^{10}$ (a statistic varying between 0 and 1 , measuring $\frac{m-1}{n-1}$ where $m=$ position and $n=$ size of sibship, with a mean of 0.5 in the general population), was $0.54(0.07)$. The mean (SD) age of the mothers was $35.0(4.5)$ and of the fathers was $36 \cdot 7$ (4.9). Daytime wetting had been present continuously from infancy in 34 instances. Associ- 
ated bed wetting was found in 20 and faecal soiling in five.

The trial was approved by the district ethical committee.

Paediatric assessment. All children were seen with a parent by one paediatrician (SRM). A full clinical history was taken before the child was physically examined. A sample of urine was collected for examination and culture. Intravenous urograms were taken in 42 children and a voiding cystourethrogram in 41 (all those with previous or present urine infection had both investigations).

In addition, all the children had urine samples checked at monthly intervals, at the time of any suspicious symptoms, as well as a few days after the conclusion of a course of antibiotics. (Any appreciable bacteriuria was eradicated with a three day course of antibiotics. Recurrence of infection was treated similarly and then by prolonged low dose prophylactic treatment, usually in the form of nitrofurantoin $25 \mathrm{mg}$ every night).

Of the nine boys, two were considered to have had previous urine infections. None had bacteriuria during the study that required chemotherapy. Of the 35 girls, 21 were considered to have had previous urine infections and 12 had one or more episodes of infection during the study and eventually required continuous prophylactic treatment.

Of the 42 intravenous urograms, 35 yielded normal results. Four showed duplex systems on one side, two entering the bladder by two ureters and two by a single ureteric opening. Two children had mild clubbing of the calyces and cortical thinning, suggesting 'pyelonephritis', which was bilateral in one and unilateral in the other. One child had what seemed to be a dysplastic kidney on one side.

Of the 41 cystourethrograms, 25 yielded normal results. Twelve showed vesicoureteric reflux, which in five was minor (grade 1), but four showed grade 2 and three grade 3 reflux. In all but two (which showed bilateral grade 2 reflux) the reflux was unilateral. Three cystograms showed paraureteric bladder diverticula and one a mildly trabeculated bladder.

Psychological assessment. When the preliminary paediatric assessment had been completed the mother and child were seen by a psychologist (SH), who described and explained the trial. The contingent alarm that went off when wetting occurred might help the child to recognise when the bladder was full. The non-contingent alarm that went off from time to time, irrespective of wetting events, would remind the child to use the toilet and might help build up more regular toilet visiting habits. It was arranged for child and parents to chart wetting and soiling by checking four times a day and to note visits to the toilet for a month before treatment was started. The psychologist told the mothers that she would keep in touch by home visits and, when possible, by telephone as well as during their regular visits to the outpatient clinic. General advice was given about personal hygiene, the use of bactericidal powders and deodorants, and the need to empty the bladder regularly, particularly at school (free access to toilets was facilitated by contacting the teachers).

About 10 days later the psychologist interviewed mothers at home, asking about features of the wetting problem and home circumstances. The Rutter A scale was used, which provides a standard set of questions for asking mothers about the psychological adjustment of their children. ${ }^{4}$ Tape recordings of this part of the interview were made. Mothers completed a self administered dependency questionnaire $^{5}$ concerning the child and a Leeds scale with regard to their own psychiatric state. ${ }^{6}$ Children were seen on their own to explain the procedure. Subsequently, class teachers were asked to complete the Conners teachers questionnaire. They were also asked to fill in one of these scales for the next child of the same sex on the class register for us to use as a control subject. Mothers of these control children were asked to complete Rutter A, Leeds scale, and self administered dependency questionnaire forms. When children and parents had agreed to try using the alarm at school as well as at home teachers were asked to allow the child to leave the class when the alarm sounded, under the pretext of having to take medication, so that they could change and use the toilet. A month after the initial contact and immediately before using the alarm the child was brought back to hospital for a video recorded observation session as previously described. ${ }^{8}$ This provided information about the circumstances surrounding the occurrence of wetting and urges to use the toilet as well as an estimate of maximal functional bladder capacity. (Halliday S, Berg I, Meadow SR. In preparation.)

Reliability of ratings of disturbance. There was complete agreement in $70 \%$ of instances when tape recorded interviews with mothers were rated for the presence of psychiatric disturbance in the children by three independent researchers (IB, $\mathrm{SH}$, and $\mathrm{Dr}$ $\mathrm{G}$ Richardson). Discrepancies of more than one point on a three point scale (normal, borderline, or disturbed) only occurred twice. There was total disagreement between the researchers on only three times. Agreement between pairs of researchers ranged between 74 and $84 \%$. 
The alarms. The two alarms (Headingley Scientific Instruments*) were identical in appearance and were worn in the same manner. A flat plastic wetting sensor, silvered on one side, was attached to underpants by sticking plaster. Wires led from the sensor to a small flat box, which contained the alarm mechanism, worn on a belt or in a pocket. When the buzz occurred, because urine had come into contact with the sensor in the case of the contingent alarm or automatically in the case of the non-contingent alarm, the alarm could be turned off by unplugging the lead. Plugging the alarm in again reset the device. The child was instructed to go to the toilet to pass water when the alarm went off, to change any wet pants, and then reset the buzzer.

Compliance measures. A calibrated capillary tube containing mercury was fitted inside the box worn by the child. The mercury level rose at a constant rate when the alarm device was switched on, taking 100 hours to reach the end of the tube. Its position was recorded two weeks and one month after treatment began.

Quota allocation system. After wetting events had been recorded for a month without any treatment (the initial four weeks baseline period) children were randomly allocated to one of the two types of alarm. This was achieved using a quota allocation system, ${ }^{9}$ allowing for sex, age group (5-8, 9-12, and 13-16 years), type of daytime wetting (primary or onset), associated nocturnal enuresis, associated soiling, frequency of diurnal enuresis (up to twice a week, three to six times a week, or daily) as well as psychiatric disturbance (definite, borderline, or none). A secretary carried out the allocation and the research workers were not told which type of alarm the child was using. Any device that failed to function satisfactorily was replaced by the secretary so that the psychologist and paediatrician remained in ignorance of the type of alarm allocated.

Criterion of success. Success was taken to be six consecutive weeks without daytime wetting. When wetting continued treatment with the alarm was stopped after three months and management was considered to have been a failure. The only exception was when a urinary tract infection had been found and treated. An additional fortnight using the alarm was then allowed on each occasion infection occurred.

Assessments after treatment. Within a month of

*43 Westcombe Avenue, Leeds LS8 2BS. stopping treatment Rutter A scale, self administered dependency questionnaire, Leeds scale, Conners teachers questionnaire, and the observation under water load conditions were repeated. Recording of wetting events was stopped. Outcome was assessed one month after stopping treatment.

Relapses. Two consecutive days on which day wetting occurred was considered a relapse. When this happened the family was asked to arrange for the alarm to be used for a week.

Follow up. A questionnaire, seeking information about wetting events, voiding habits, occurrence of urine infection, and the child's behaviour and health, was sent to parents by post at three and six months and one and two years after completion of treatment. (The questionnaire is available on request from the authors.)

\section{Results}

Over the four week baseline period the mean (SD) number of daytime wetting events was $38(20)$. The mean (SD) functional bladder capacity was 160 (75) $\mathrm{ml}$. Assessments of psychiatric disturbance in the children by a child psychiatrist (IB) from listening to tape recordings of interviews with mothers conducted by the psychologist suggested that 11 children $(25 \%)$ were definitely, and six possibly, disturbed emotionally. On the Leeds scales, which estimate adult psychiatric disturbance, mothers of the daywetting children were found to have a mean (SD) score of $3.8(3.7)(n=38)$, which was significantly higher than that of the mothers of controls, which was $2 \cdot 2(2 \cdot 0)(n=34)(p<0 \cdot 02)$. Their mean (SD) A score of $4 \cdot 1$ (3.5) did not differ significantly from that of the controls, which was $3 \cdot 5(2 \cdot 6)$.

No significant differences emerged between day wetters $(n=44)$ and controls $(n=34)$ on the four subscales of the self administered dependency questionnaire concerned with dependency of child on mother. There was evidence from the Conners teachers questionnaire that the 42 day wetting children showed more disruptive behaviour (factor I mean (SD) 0.2 (SD 0.4), inattentiveness (factor II mean (SD) $0.7(0 \cdot 7)$ ), and overactive restless behaviour (factor IV mean (SD) $0 \cdot 8(0 \cdot 7)$ ) than the 30 controls (factor I mean (SD) $0 \cdot 1(0 \cdot 1), \mathrm{p}<0.05$; factor II mean (SD) $0 \cdot 3(0 \cdot 4), p<0 \cdot 01$; and factor IV mean (SD) $0.4(0 \cdot 4), p<0 \cdot 01)$. A significant excess of antisocial behaviour was evident in 22 day wetters tested on the Rutter A antisocial subscale (mean (SD) $1.8(2)$ ) compared with 19 controls (mean (SD) $0 \cdot 5(0 \cdot 8), \mathrm{p}<0 \cdot 01)$. No significant differences in 
neurotic behaviour between daytime wetters and controls emerged.

None of the measures made before the start of treatment revealed any significant differences between children in the contingent $(n=22)$ and the non-contingent $(n=22)$ alarm groups. (Analyses of variance were used for comparisons of quantitative variables and Fisher's exact tests for looking at qualitative variables; $p<0.05$ was taken as the significance level).

Immediate response. Five children, three with the non-contingent and two with the contingent alarm, dropped out of the study during the first week in which they were supposed to use the alarm. There were 13 successes with the non-contingent alarm, a $59 \%$ success rate if the three initial dropouts are counted as failures and a $68 \%$ success rate if these dropouts are disregarded. There were 16 successes with the contingent alarm, success rates of $73 \%$ and $80 \%$, respectively. The greater success rate of the contingent group, whether dropouts were considered failures or not, did not reach significance using Fisher's exact test. Of the total group of 44, 29 $(66 \%)$ became dry, five $(11 \%)$ dropped out, and 10 $(23 \%)$ were still wetting during the day at the end of the trial. For those who persisted with treatment for longer than a week, $75 \%$ were successes.

Considering improvement in terms of median percentage change from the degree of wetting during the baseline period, week by week during the three months of the trial, it was found that those who eventually became dry had a fully dry week by the sixth week of treatment. No child who ultimately failed achieved this (Figure).

Change in daytime wetting during the trial on a seven point scale, varying from $1=$ more than $85 \%$ worse to $7=$ more than $85 \%$ better, was used as an outcome measure in carrying out a multiple regression analysis, using age of child, social class, compliance with treatment, history of urinary tract infection, and baseline day wetting as predictors. A stepwise method was employed. The only significant predictor of outcome was severity of day wetting in the month before treatment, particularly the number of wetting events that were recorded by the third week of the baseline period $(\mathrm{F}=12.1, \mathrm{p}<0.001$, $r=0 \cdot 5)$. When improvement was equated with $75 \%$ less wetting during the trial, and 30 wetting events by week 3 of baseline assessment was called 'severe wetting', then 16 of 20 'less severe' cases improved and nine of 19 'severe' cases improved; this difference was significant $(p=0 \cdot 05$, Fisher's exact test two tailed).

The mean (SD) number of weeks needed to reach the remission criterion-that is, six consecutive weeks of being dry by day-was 18 (8), with no significant difference between treatment groups. Most of the children complied with the instructions to use the alarm; only four children failed to show at least 100 hours of alarm use (as verified by the mercury column used to estimate compliance) in the first month of using the alarm. None of the scores on the various scales used to estimate psychiatric problems in mothers or children changed significantly in the day wetters or controls after treatment,

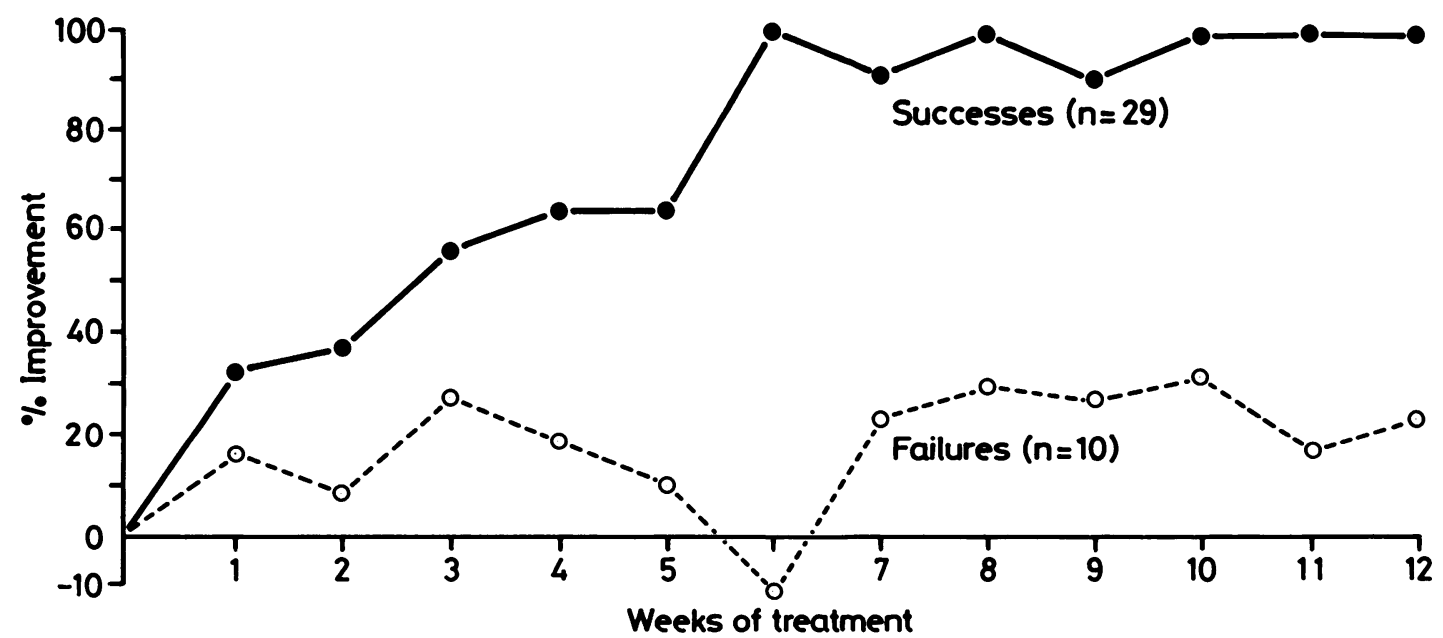

Figure Median percentage change in wetting from baseline treatment period over three months in 39 children with daytime wetting treated with an alarm. 
taking the groups as a whole. The only exception was a significant fall in the assistance subscale of the patient on the self administered dependency questionnaire after treatment (mean decrease 0.9, $\mathrm{t}_{\mathrm{corr}}=2 \cdot 3, \mathrm{p}<0.03$, from a pretreatment average (SD) of $7 \cdot 5(2 \cdot 7), n=44)$. When those who successfully responded to treatment were looked at separately, however, not only was there a significant fall in the assistance subscale of the questionnaire (mean fall $0 \cdot 9, t_{\text {corr }}=2 \cdot 1, p<0 \cdot 05$ ) but scores on factors I (mean fall $2 \cdot 0, \mathrm{t}_{\mathrm{corr}}=2 \cdot 8, \mathrm{p}<0 \cdot 01$ ) and IV (mean fall $2 \cdot 0, t=2 \cdot 9, p<0 \cdot 01)$ of the Conners teachers scale also showed significant reductions. In those who failed to respond to treatment numbers were small but no change in assistance subscale of the self administered dependency questionnaire was evident, factor I of the Conners teachers questionnaire showed an increased score (mean 1.0, $\mathrm{t}_{\mathrm{corr}}=2 \cdot 5, \mathrm{p}<0 \cdot 03$ ), and the mothers' mean Leeds D scale score declined significantly (mean fall $2 \cdot 1$, $\left.\mathrm{t}_{\mathrm{corr}}=2 \cdot 9, \mathrm{p}<0 \cdot 02\right)$.

Ratings of childhood disturbance calculated by the psychiatrist from recordings of interviews with mothers carried out by the psychologist suggested that seven of the children were definitely and seven possibly disturbed psychiatrically. Of the 25 children who were not considered to be disturbed before treatment, 24 showed no change. Six of the 14 who had some degree of disturbance beforehand remained unchanged, whereas the other eight had improved, with five being completely free from disturbance. Treatment groups did not differ in these respects.

The mean (SD) functional bladder capacity after treatment was $176(108) \mathrm{ml}$. Change from mean bladder capacity measured before treatment began was not significant. Considering only those children who had no urinary tract infections while using an alarm, however, there was a significant rise in mean (SD) bladder capacity with treatment (before treatment $146(68) \mathrm{ml}$ and after treatment $180(107) \mathrm{ml}$, $\mathrm{p}<0 \cdot 01)$.

Follow up. The 29 children who responded successfully to treatment were followed up after six months. Two were found to have relapsed, so that $27(93 \%)$ were still free from day wetting. After a year only 25 could be reviewed and $19(76 \%)$ remained dry by day. Twenty two children were followed up at six months and one and two years after treatment. Of these, $18(82 \%)$ had remained free from day wetting throughout, although one had had a temporary relapse during a bout of influenza.

\section{Discussion}

The findings of the trial were encouraging in that about two thirds of children with daytime wetting responded satisfactorily to either alarm. This is a considerable improvement on previous methods of treatment. Our previous investigation showed no significant difference between the drug imipramine and a placebo, in the management of diurnal enuresis, with only a fifth of the children becoming reliably dry. ${ }^{3}$ There is some evidence that mothers of daytime wetters attribute the problem to their child's reluctance to go to the toilet in time, ${ }^{11}$ and this may well be a factor, ${ }^{8}$ so that effectiveness of the non-contingent alarm may lie partly in persuading the child to empty the bladder regularly when it is not too full and also doing this without prompting by parents.

The non-contingent alarm is simpler to use than the contingent one. The finding that it worked just as well as the contingent one, within the limits of a reasonably sized clinical trial, suggests that little would be lost in using it routinely for all children with diurnal enuresis. Our current clinical practice is to use a non-contingent alarm in the form of an inexpensive commercially produced timer, which is less cumbersome and more robust than the alarm used in the trial. Staff trained in using such a noncontingent alarm provide the family with the sort of guidance and support that was given during the trial. It is also arranged for the school to permit the child to leave the classroom to use the toilet more easily than is normally allowed. (During the trial no adverse reports of using the alarms in school were received from teachers.)

The design of the study did not permit the conclusion that the use of the alarms per se brought about improvement in the day wetting. The improvement might possibly have been due to placebo effect and support given by the researchers. This seems rather unlikely as poor results were obtained in our previous drug trial at the same clinic ${ }^{3}$ and successful outcome was not related to frequency of contact with the psychologist in the investigations reported in this paper. (None of the failures had less than three or more than six visits (mean $4 \cdot 1$ visits) and the successes had between one and five visits (mean 3.4 visits).

It was encouraging that only about $10 \%$ of patients dropped out, rather better than when bedwetting was treated with a pad and bell. ${ }^{12}$ Although about half of children did not wear their alarm all the time, particularly at school, this did not seem to influence the results, and compliance measured in terms of hours of usage was generally good. The finding that severity of daytime wetting was identified as the principal antecedent of poor response to treatment was not unexpected. The failure to find any connection between relief of 
daywetting and improvement of bedwetting is in keeping with previous work. ${ }^{2} 3$ What seemed to be an effect of urinary tract infections in preventing a significant increase in the maximum functional bladder capacity after treatment was interesting and requires further investigation.

The high incidence of urine infection, particularly in the girls, fitted in with our previous studies of children with day time wetting." It seems that wetting during the day predisposes to infection and that infection itself makes bladder control more difficult and day time wetting more likely. Bladder detrusor instability is more pronounced when the urine is infected. Thus investigation of urine for infection and then elimination of that infection is an integral part of the management of day time wetting. The high incidence of minor radiological abnormalities of the urinary tract was surprising. The abnormalities themselves, however, were probably not responsible for the wetting. The radiological abnormalities seemed to be more in the nature of evidence of functional bladder disturbance, perhaps signifying that anatomical and functional development of the urinary tract went slightly astray earlier in life.

The high level of psychiatric problems in the sample was not surprising as this is a well established feature of diurnal enuresis. ${ }^{13-15} 1$ The finding that mothers of daywetting children were more likely to be depressed, as indicated by high scores on the Leeds scale, was interesting as measures of maternal psychiatric state have not usually been used in previous studies. ${ }^{16}$ Also the use of a dependency scale provided new information about daytime wetters. It is interesting that there was in fact no evidence that children who wet by day were deviant in their dependency on mother. It is also noteworthy that the psychiatric disorders of the day wetting children were more in the nature of antisocial than neurotic disturbance by comparison with previous studies of a similar kind, ${ }^{3} 11$ considering the preponderance of girls in the sample of cases. Changes in the average scores on the various scales after treatment provide some indications of possible processes involved in remission. The increased independence of the children, who were relieved of daytime wetting, in respect of 'task oriented' dependency ${ }^{5}$ and the reduction in antisocial conduct suggest that the children who recovered became more cooperative and were more prepared to look after themselves. The small number of failures in treatment make comments about changes in questionnaire measures in that small group difficult.

The Mental Health Foundation provided a grant to enable SH to work on the project. We thank Mr R McGuire for advice about the use of his quota allocation procedure. Dr G Richardson for rating tape recordings of interviews, Mr K Yates for helping with the videotape recordings of the observation sessions, and Mrs Anne Maric Brabiner. Mrs Patricia Mullins, and Mrs Wendy Pearson. who prepared the manuscript.

\section{References}

1 Berg I. Day wetting in children. J Child Psychol Psychiatry 1979;20:167-73.

2 Fielding D. The response of day and night wetting in children and children who only wet at night to retention control training and the cnuresis alarm. Behav Res Ther 1980:18:305-17.

${ }^{3}$ Meadow SR, Berg I. Controlled trial of imipramine in diurnal enuresis. Arch Dis Child 1982:57:714-6.

+ Rutter M. Tizard J. Whitmore K. Education, health and behaviour. London: Longmans, 1970.

5 Berg I. A self-administered dependency questionnaire (SADQ) for use with the mothers of school children. BrJ Psychiatry 1974:124:1-9.

"Snaith RP. Bridge GWK. Hamilton M. The Leeds scales for the self-assessment of anxiety and depression. $\mathrm{Br} J$ Psychiatry 1976:128:156-65.

7 Conners CK. A teacher rating scale for use in drug studies with children. Am J Psychiatry 1969:126:884-8.

* Fielding D. Berg I. Bell S. An observational study of posture and limb movements of children who wet by day and at night. Dev Med Child Neurol 1978:20:453-61.

"McGuirc R. Quota allocation. J Psychosom Res 1968:12:168-9.

10 Slater E. Birth order and maternal age of homosexuals. Lancet 1962:i:69-71.

"Berg I. Ficlding D, Mcadow SR. Psychiatric disturbance, urgency and bacteriuria in children with day and night wetting. Arch Dis Child 1977:52:651-7.

12 Young GC. Morgan RTT. Childhood enuresis: termination of treatment by patients. Community Med 1972:129:247-50.

1.3 De Jonge CA. Epidemiology of enuresis. In: Kolvin I. MacKeith RC. Mcadow SR, eds. Bladder control and enuresis. London: W Heinemann, 1982.

it Hallgren B. Enuresis II: a study with reference to certain physical, mental and social factors possibly associated with cnuresis. Acta Psychiatrica et Neurologica Scandinavica 1956:31:379-401.

15 Hallgren B. Enuresis: a clinical and genetic study. Acta Psychiatrica et Neurologica Scandinavica (Suppl) 1957:32:114-9.

in Shaffer D. Enuresis. In: Rutter M. Hersov L. eds. Child and adolescent psychiatry. 2nd ed. Oxford: Blackwell Scientific Publications, 1985:465.

Correspondence to Professor S R Meadow. Department of Pacdiatrics and Child Health. St James's University Hospital. Leeds LS9 7TF. England.

Received 8 August 1986 